\title{
CRITERIA FOR SUBSPECIES, SPECIES AND GENERA, AS DETERMINED BY RESEARCHES ON FISHES
}

\author{
By \\ Carl L. Hubbs \\ University of Michigan, Ann Arbor, Michigan
}

The criteria for the minor taxonomic groups of fishes do not differ essentially from those that are pertinent to the subspecies, species and genera of the tetrapod classes. The increasingly detailed and penetrating researches of recent years keep emphasizing the conclusion that these categories are of essentially the same sorts in all groups of vertebrates. Speciational processes appear to be similar throughout the Vertebrata, in fact throughout most of the organic world.

For several reasons, however, we find that fishes are particularly well suited to an analysis of the minor taxonomic categories, and hence to an appreciation of the criteria by which these assemblages can be recognized. In the first place, fishes are rich in differentiae: they fairly bristle with diagnostic external features, and characters penetrate their whole anatomy (the internal characters may readily be investigated, for the whole bodies-not just skins-are preserved). Most of the distinguishing features are readily subject to precise evaluation, and hence to statistical analysis; meristic differences are common. For such studies it is often possible to make use of hundreds or even thousands of specimens, for large series of fish are readily collected and preserved. The critical relations between individual and racial modifications are particularly amenable to study in fishes, for the aquatic vertebrates are molded by their environment to a greater degree than are the representatives of the so-called higher groups; the trend of vertebrate evolution has been to free the organism from domination by its physical environment. The fact that many of the stocks or races within a fish species occupy highly diverse ecological situations often makes it possible, without recourse to experiment, to analyze the individual modifications and the genetic responses to the environment. Finally, the genetic basis of characters and the genetic interrelationships of forms can be determined more readily by experiment in the Pisces than in most other groups, because many fishes may readily be propagated, and because of the frequent occurrence, in certain fishes, not only of subspecific intergradation, 
but also of interspecific and even of intergeneric hybridization. Natural hybrids between species seem to occur more commonly in fishes than in any other group of animals. Fishes are especially well suited to the study of forms as populations of living animals, not merely as samples of preserved specimens.

Except as they may be arbitrarily erected, the distinguishing criteria cannot be more precise than the true nature of the taxonomic categories. Every advance in the general systematics of fishes tends to dispel further any idea that these categories differ from one another with sufficient uniformity to permit lines of clear demarcation to be drawn consistently between them; or that any of the assemblages are subject to a very precise and exclusive definition. He who creates hard and fast distinctions between the systematic categories is merely drawing lines of chalk down a blackboard.

Largely on the basis of convention, we recognize a primary distinction between kinds or forms (colonies, subraces, races, subspecies, and species) on the one hand, and groups (species groups, subgenera, genera, and larger categories) on the other side. The kinds are regarded as the units which make up the groups, but each kind may ordinarily be shown to be a group comprising lesser units, down to the small colony and finally to the individual (which in turn is an integrated colony). And each minor superspecific group, such as a genus, may be scarcely less a kind than is the species; this is most obviously true of monotypic genera. It is therefore difficult to state criteria, even for the distinction of kinds from groups. All kinds of animals and all of the groups are surely to be regarded as graded levels of evolutionary differentiation.

Races, subspecies and species are all regarded as kinds or forms, and therefore as having common terms in their definition. Kinds may be defined as self-perpetuating populations with a considerable degree of uniformity in time and in space. There are then three essential considerations in the concept of animal kinds: genetic basis; integrity as populations; success in the struggle for existence. Somatic responses of the individual are not true forms. Systematic characters must have a genetic basis; if this is not demonstrable, it is to be inferred. But one or a few genetically distinct animals do not necessarily constitute a systematic kind. There must be a population, self perpetuating and distinctive. Animal kinds are not made up of characters, or of museum specimens, nor of age or sex variants, nor of interbreeding phases; they are living entities in a living world. And, as David Starr Jordan has insisted, populations with a genetic basis do not qualify as species (or as infraspecific units) until they have successfully run the gauntlet in the 
vigorous struggle for existence in nature. In speciation, survival is quite as significant as genetic potential.

The fish data seem particularly valuable in the classification of the types of animal kinds. In addition to the usual distinction of species and subspecies, the race is interpreted as a distinct taxonomic category by most ichthyologists. Unlike many ornithologists and mammalogists, they do not use "race" or "geographic race" as a synonym of subspecies. For several reasons, such as the abundance of available material and the demand for the population-analysis of commercial fishes, minor races have been studied more in the Pisces than in other groups. Ichthyologists have therefore had most reason to separate the race as a category distinct from subspecies. They think the distinction to be a valuable one and urge its general adoption. Such studies as those of Sumner and of Dice on Peromyscus and of Miller on Junco suggest that the concept of the race, as a lesser division than the subspecies, will come into wide use through the vertebrates.

Races, in the sense here advocated, are not accorded a place in the current system of zoological nomenclature. One reason is that since such races are ordinarily distinguishable only by average characters that may call for statistical treatment, the routine identification of single specimens or small samples would of ten be difficult or even impossible. Race ranking may be accorded forms, like local types of Gasterosteus aculeatus, which are so confusingly numerous or so complex in characters, and so complicated in genetic and geographical relationship, as to transcend any ordinary scheme of zoological nomenclature. Excluding the races from the nomenclatorial system does not imply that their distinction and study are unimportant. On the contrary, the minor races-potential species in the early stage of their making - are perhaps the most significant material for the study of speciation.

For one, I decry, as impracticable and as contrary to the International Rules, the use of a quadrinomial system for the designation of races, and I still more vehemently oppose the nomenclatorial recognition of "morphae" and "nationes," as distinct from subspecies.

Unless the systematics are excessively complicated, I would designate as a subspecies any genetic form which shows reasonable geographical or ecological consistency, and which can usually be distinguished on its totality of characters. Ordinarily it would be required that much more than half of the given population be distinguishable; not necessarily at all times and places, but at least in one sex, at some given stage of development. Interpreting the ensemble of characters, as by the method 
of the character index, may aid in defining a form as a subspecies rather than as an unnamed race.

Unlike races, subspecies are animal kinds which are sufficiently clearcut as to be thought worthy of a place in the nomenclatorial system, but which do not give evidence of being completely differentiated. Species, in simple terms, may be denoted as kinds (defined above) which are completely differentiated from all others, but which do not themselves include fully differentiated subdivisions. These definitions of the categories of animal kinds are based on practical as well as theoretical considerations. Recent studies in ichthyology seem to justify the compromise.

Despite the obvious intergradation between systematic categories in fishes, ichthyologists share with other systematists the view that a higher degree of distinctiveness and reality is possessed by the species, than by any infraspecific or any supraspecific category. However diverse our opinion may be as to the magnitude or consistency of this distinction, or however much we may confuse the issue by philosophical quibbling over "reality" versus "concept," we agree in the view that the species represents a rather definite and relatively stable level of attainment in evolution.

The creation of a new species marks the approximate end of the speciational process, for differentiation has then proceeded to completion or nearly so. Species, therefore, are usually trenchantly distinguished from one another. The ichthyological evidence leads us to suspect that species dominate the systematic picture, not only because of their distinctness but also because of their long life. Once the specific level has been attained, with its more or less complete genetic segregation, further divergence will probably be accelerated, but the local and individual variants that appear will ordinarily long remain an interbreeding part of the species complex. The further transformation or division of the species into one or more new species is a hurdle which Nature finds difficult and time-consuming to jump.

In my younger, more radical, years I was so much impressed with the view that species are the most natural and distinctive of the taxonomic categories, that I considered proposing a uninomial system of zoological nomenclature. Such. a system, it was thought, would have many advantages over the Linnaean one which has been in use for nearly two centuries; it would indeed divorce taxonomy from nomenclature, lead to the stability, uniformity and brevity of names, and force precision in identifications; and it would be the logical outcome of the tendency 
toward monotypic genera. If subspecies were always sharply distinct from species, such a scheme would indeed have a strong call for adoption.

However, the more intensely I have studied fish species, the greater the number of species I have examined, and particularly the more thoroughly I have studied whole species groups throughout the range of the complex, the more difficulty I have found in the interpretation of forms as subspecies or as species and in distinguishing species from species groups: The distinctions appear more and more arbitrary. This realization, along with the attainment of a more conservative age, has led me to abandon the idea of proposing a new system of nomenclature.

Possibly the fresh-water fishes (of which I have studied more than a million specimens) are peculiar in the high percentage of forms which only by arbitrary decision can be classed as subspecies or as species. On the other hand, marine fishes and other groups of vertebrates may seem to exhibit sharp distinctions between species and other taxonomic categories, only because the systematic studies have been based on fewer specimens or have been less analytical, or because the systematic picture has been more dominated by convention and "authority." I suspect that the truth involves both alternatives.

Incompleteness versus completeness of differentiation is the main test by which subspecies may be distinguished from species, on the kinetic concept here advocated. Degree of differentiation is difficult to determine, especially from small samples, but is the truest measure we can obtain of the stage of speciation. No other criterion would seem to have so sound a speciational basis, or to be so consistently applicable.

Determining or predicting whether two kinds do or do not intergrade therefore becomes a matter of paramount nomenclatorial importance. Intergradation is a central problem in practical systematics as well as in speciational research.

Intergradation would be rather easy to demonstrate, if it were regularly of the simple type which alone has been recognized by many vertebrate zoologists. According to their oversimplified view, intergrading forms have well-defined ranges which are separated by a narrow belt in which the characters of the one subspecies grade rapidly and evenly into those of the other. Recent variational analyses by ichthyologists, however, keep emphasizing the views: (1) that this simple, narrow-band type of intergradation is perhaps the exception; (2) that there are many, often complex types of intergradation; and (3) that, in the area of intermixture, there is every stage between complete fusion and almost complete genetic isolation. Such critical studies as those of Blair on Bufo, of 
Fitch on Thamnophis, of Miller on Junco, and of Dice on Peromyscus, confirm the view that varying patterns and degrees of intergradation also characterize the higher vertebrates.

The simple intermediate-band type of transition between well-defined subspecies of fish does undoubtedly occur, especially in some forms with coast-wise distribution (for instance in certain West Coast blennies). At times, the area of intergradation may be very broad, greater than the range of either subspecies. Or the area may be small, or may be a longitudinal connecting strip rather than a transverse band. Commonly there is a chain of forms, or a rather even gradation from one end of the range of the species to the other. Among the fresh-water fishes intergradation tends to follow what we have termed a mosaic pattern.

The ranges of two subspecies are commonly separated by a wide belt, in any part of which we may find: (1) entire populations typical of either form; (2) mixtures of the two types; (3) a complex of either one or both types plus intergrades; or (4) intergrades alone (Notropis cornutus chrysocephalus and $N$. c. frontalis, for example, intergrade in all these ways). If we free ourselves of preconceived ideas of how subspecies are or should be interconnected, we can readily appreciate why such complex patterns are probably the rule. Two forms which occupy distinct territories, with different climatic conditions, will almost surely develop different environmental responses. For this reason the two types, in the intervening zone, will tend to segregate themselves, or, if they occur together, they will often spawn in different niches or at separate times. Where the environmental conditions are contrasting, the two types will tend to maintain their identities; where the environment is uniform, the forms will intergrade.

Such diverse types of intergradation will probably be found to hold throughout the vertebrates. To treat as subspecies only those forms which intergrade in the conventional pattern would be indefensible.

The common tendency, at least in fresh-water fishes, for two forms to intergrade in some localities but not in others, renders difficult and equivocable the test that is most commonly applied, usually without doubts or reserve, to determine whether two forms should be separated as subspecies or as species. This is the test of determining whether two forms that live together do or do not intergrade. Further difficulties in applying this test arise from the incompleteness of available data, and from the subjectiveness that must ordinarily be involved in reaching an "inference" concerning the genetic relationship; from the circumstance that the really critical data pertain to the exact time and locale of breeding; and from the circumstance that two forms may occur together with- 
out interbreeding, yet be connected by a chain of interconnected subspecies (the "open circle").

Then, too, we encounter the uncertainty, that forms morphologically intermediate between two kinds may arise through the interbreeding of these two kinds. The interjacency of characters may be due to chance variation, or to independent adaptations to intermediate conditions. On the subspecies concept that requires intergradation as a result of interbreeding, such interpretations would throw the systematic decision into doubt. In my view, however, the existence of truly intermediate types would be taken as evidence that subspecies are involved, even though the intermediates had not arisen through interbreeding. I would not even demand that the intermediates occupy an intermediate range: they may crop out at any interior, peripheral or even disconnected location.

In general I subscribe to Jordan's Law, that nearest relatives tend to occur in adjacent, rather than in the same or in distant territories. I therefore regard geographically contiguous but complementary ranges as typical for subspecies. It would, however, be unwise to accept this relation as a criterion for subspecies. Forms otherwise like subspecies, and regarded by me as such, may occur in widely separated regions, or on narrowly but completely separated ranges (as in adjacent stream systems tributary to the ocean). Again we find forms of the subspecies type whose ranges are entirely enclosed within that of their cognates: thus Boleosoma nigrum eulepis exists in appropriate pockets within the range of $B$. n. nigrum, with a halo of intergrades around each of the units of discontinuous distribution.

In increasing number, subspecies in fishes are being shown to be ecological (or microgeographical) forms, which occupy diverse habitats in the same or in very broadly overlapping areas. They exhibit a pattern of partial or complete intergradation, in which the intergrades occur mosaically at appropriate points throughout the common range of both forms. This type of subspeciation is proving to be common in fishes, notably among the fresh-water fishes of the western United States. Notropis volucellus is an outstanding example in eastern North America. Most geographical differentiation is in part ecological, and most ecological subspecies have some geographical basis. The two types completely intergrade. Obviously, then, the criterion of intergradation is not to be restricted to purely geographical interconnections.

Not only may subspecies violate the rule of adjacent, complementary ranges, but full species may show the distributional type that is more typical of subspecies. Many unquestionable species-even some genera 
-of fish show complementary distribution. It could hardly be expected that geographically seriated subspecies would never differentiate in situ into seriated species.

Character intergradation between two wholly or almost wholly isolated (non-interbreeding) populations is not, in my opinion, to be excluded as evidence of incomplete differentiation, and may therefore be taken as a criterion of the subspecific status of the two kinds. It is probable that some of the intergradation that is observed between forms of overlapping or contiguous ranges is due to parallel adaptations, of separate origin, to the intermediate conditions, or to chance variation rather than (or in addition to) interbreeding. The criterion for subspecies, of intergradation due to cross-mating only, would seem to be impracticable as well as illogical.

Thus I regard intergradation of almost any type as evidence that speciation is not complete and that the forms involved are on the subspecies level of differentiation. I would, however, lay much greater stress on actual intergradation in nature than on potential interbreeding. I do so not only because I lean quite as heavily on the survival as on the genetic aspect of the concept of animal kinds, but also because it is more objective to deal with what exists than with what we may infer to be possible. The curse of most systematic work has been its subjective or "authoritative" basis, against which we should react. What actually occurs seems to be most significant, and it is generally admitted that many apparently "good" species of fish and other animals interbreed freely in captivity yet never or very seldom do so where they occur together in nature. There are other than purely genetic isolating mechanisms. As Haldane has written, "The physiological barrier between two species may be of several different kinds. It may occur before or after fertilization, and the hybrids, if any, may be sterile, or more or less completely fertile." I prefer to regard as full species any two completely distinct forms which do not intergrade in nature, whether or not they fail to interbreed because of their isolated ranges. If under appropriate circumstance the two forms at any time come together and interbreed regularly, I would take this as evidence that they have reverted from the specific to the subspecific category of differentiation.

Thus I regard it as impracticable to restrict the concept of subspecific intergradation to connections between forms that are assumed to be diverging; that is, to what may be called primary or antecedent intergradation. It will commonly be difficult or impossible to determine whether the intermediate characters of a given population date back to the initial divergence of the two types, or are due to the secondary or 
subsequent meeting and interbreeding of populations, or of entire forms, which had previously been isolated for some time. Our analyses of intergrading forms lead us to believe that most intergradation, even in a single region, is due to a combination of the primary and the secondary processes. It would seem wholly impracticable to set up divergence, as opposed to convergence or fusion, as a criterion of the subspecific level.

It would also be unwise to restrict the concept of subspecies to forms which change as organismic units from one to another across their zone of intergradation. An increasing number of cases are being discovered, in fishes as well as in other groups, in which the units of geographical variation and intergradation are characters which do not all change at the same region. Thus, as we proceed southward, a color feature (for example) may intergrade at one point, whereas a scale character will change farther south. If the intervening type with the color of the southern race but the squamation of the northern one has a considerable integrity in characters and in range, it may be recognized as an intermediate subspecies. The same treatment may be accorded intermediate populations which exhibit harmonious or concurrent intergradation in all characters. Whether or not to recognize the intervening subspecies should depend on individual and geographic consistency in characters.

Furthermore, the two sexes may intergrade at different points.

Commonly the specific level is evidenced by completeness of differentiation in each of several respects, usually in at least one character. Complete differentiation, however, may be demonstrated only when the ensemble of characters is studied. Each differential feature when considered alone may show an overlap, when the frequencies for the two types are compared. Nevertheless, the kinds may invariably be distinguished on the basis of the ensemble of their characters. This completeness or near-completeness of differentiation may often be statistically demonstrated by the use of the character-index method which we have been employing.

We do not often hear, in modern ichthyology, of the formerly common expression, "specific characters," as something essentially different from "varietal characters" or from "generic characters." Consistency is a better test than kind for the taxonomic significance of a character. Thus we find that the union of the lower pharyngeal bones coupled with the transformation of conic pharyngeal teeth into molars varies considerably within a species, and in different groups of fishes characterizes a subgenus, a genus, a family, and an order-according to the current system. Characters, like gold, are where you find them.

Despite certain claims, the mode of inheritance of the systematic 
characters provides no valid criterion for systematic ranking. We can not place a given form as a subspecies or as a species, on the basis of whether its characters do or do not show sharp Mendelian segregation. Our breeding experiments with fishes and our analyses of natural hybrids are indicating that most of the many systematic characters studied, whether of race, species, or genera, behave according to a seemingly Galtonian type of inheritance, though some features of equal systematic value do Mendelize simply. This would seem to be the general rule, at least for vertebrates. Haldane wrote in 1938: "The majority of interspecific differences, however, blend, though there is usually an increased variability in $\mathrm{F}_{2}$ which can be explained as due to segregation ... The hypothesis of multiple factors is at present neither proved nor disproved."

In general, however, we find that simple Mendelian segregation is typical of "sports" and "phases" that do not occur with sufficient consistency as discrete populations to warrant their inclusion in the taxonomic system. The hereditary pigment types of Lebistes, as studied by Winge and others, and the caudal-peduncle markings of Platypoecilus, so thoroughly investigated by Gordon, I would put in the same class.

Some have held that subspecies produce intergrades uniformly intermediate in all characters, whereas species, when they interbreed, yield offspring with a mixture of the characters of the two parental types. The extensive evidence on intergrades and hybrids in fishes does not support this claim.

Evidence from reciprocal crosses between subspecies and species of fishes contradicts the odd view that "nuclear differences may account for variation within a species," whereas "the deeper differences between species depend on the cytoplasm."

In a general way only it may be said that subspecies exhibit adaptive responses to temperature and other physical features of the environment, whereas species characters, if adaptive, are correlated with food habits and other biotic factors. Such relations, however, do not hold with any great consistency and are not available as definitive criteria for systematic ranking.

There may still be some vertebrate zoologists-few ichthyologists, I hope-who would accept as a criterion for systematic ranking, the interpretation of the characteristics of the animal as adaptive or nonadaptive. Some have held that subspecific characters are adaptive, whereas specific and generic features are not of survival value; others deny that species possess the adaptive characters which the superspecific groups display. Such a criterion would be unsatisfactory, in that it would tend to maintain systematics as a subjective rather than an ob- 
jective art. I would add that adaptiveness is not rightly to be used as a criterion for systematic ranking, because, in my opinion, almost all characters-whether of races, subspecies, species, genera, or higher groups-have been involved, primarily or secondarily, in the adjustment of the animal to its particular environment.

To rank two forms as subspecies because "their intimate relationship would be concealed by a grant of specific status" seems to be an unjustified taxonomic procedure. Of course subspecies are more closely related than species, but this is true only on the average. To class a form as a subspecies by reason of one's opinion as to relationship carries all the dangers that commonly go with subjective decision. The next worker may have a different feeling. Stability of nomenclature calls for more objective criteria.

It is similarly wrong, I would say, to force a kind into the subspecies rank merely because it is a member of a minor evolutionary cluster of which the other units are subspecies. There is no reason why any member of such a group should not attain complete differentiation and hence warrant specific status.

Ordinarily an inferred monophyletic origin is regarded as a requirement for any natural group or kind. Lately some authors, notably Dice, have interpreted subspecies as ecological responses which may originate repeatedly. Such local types as the black mammals of the lava beds, or the brackish-water races of Zoarces, very probably have evolved independently in response to a like habitat. Monophylety is obviously not to be applied rigidly to infraspecific units. Until complete genetic isolation has been attained, forms will no doubt often interchange their genes. Identical or similar mutations, followed by selection, could readily transform a given kind of animal repeatedly into adaptive products that are indistinguishable or even genetically identical. The polyphyletic races of a subspecies may differentiate into distinct species; or a character which will later define a distinct species or even genus may arise independently at several localities. Hence, to some degree species and genera, as well as subspecies, may be polyphyletic.

One of the better distinctions between subspecies and species lies in the magnitude of the structural differences. But again no clear-cut, usable criterion is provided. Even within a race, terata and phases may show differences greater than those separating related genera. Some subspecies which are evenly connected by intergrades show differences which are more trenchant than many of the specific distinctions in the same group (such, for example, are the differences between Gambusia affinis affinis and G. a. holbrookii), It is to be expected that bonds of 
intergradation may be continuously retained by some forms, which have undergone an amount of differentiation that would ordinarily accompany complete speciation; or that two forms long separated and well differentiated may retain potential interfertility, so that they will again intergrade and become subspecies when their ranges come to overlap. Amount of difference is therefore not an infallible criterion for systematic ranking.

Ichthyologists will concur in the general view that genetic isolation is probably the best single index by which species may be distinguished from subspecies. Recent researches on fishes, however, prove that even this test often breaks down. Degree of fertility is positively correlated with degree of relationship, but only in a rough way. Subspecific intergrades tend to be fertile, but some crosses within a single race are sterile; except in certain groups like the Cyprinidae, most speciés are more or less completely intersterile, or produce infertile offspring, but some genera yield fertile progeny. Furthermore, in some groups there is every gradation in fertility, without the sharp distinction between interfertile subspecies and intersterile species that some geneticists, as Shull, postulate. We find this true, for example, in the genus Mollienisia, on which we have been conducting breeding experiments for ten years. Effective isolation in nature often precedes the attainment of sterility, as already mentioned in treating potential as contrasted with actual intergradation. Just where and how to draw the line between genetically connected and genetically isolated forms is often very difficult to determine in fishes, not only those which are little studied but also those which have long been subjected to breeding experiments.

\section{CONCLUSIONS}

To my knowledge no single criterion that has ever been erected will suffice to define the species, without the need for some exceptions and modifications. The more intensively species are studied, throughout their ranges, the more difficult it often becomes to decide on the taxonomic rankings. Nevertheless, most species in most groups seem sufficiently distinct to be interpreted as such by all systematists. Even when all single tests for the species level break down, a form may be recognized as a species by reason of the usual validity of a series of criteria, just as some subspecies and species may be known by the usual though not invariable possession of each of a series of characters.

Much more often than is generally supposed or admitted, the distinction of subspecies from species appears indefinite and arbitrary. Among 
the general biologists Haldane has appreciated this circumstance, for, in 1938, he wrote that: "Our general conclusion is that there is no evidence that at any rate closely related species differ in a manner qualitatively diverse from varieties." Subspecies no doubt are usually terminal twigs, but, contrary to the views of Bateson and Goldschmidt, some of these twigs no doubt grow into great limbs on the tree of evolution.

We have been surprised to find, of late, that we must be arbitrary even in ranking some forms as subspecies or as genera. This is true of two minnows of the Mohave Desert, which represent two of the wide-spread genera of the West. In the Pluvial period these fishes were obviously separated as lacustrine and fluviatile types but they are now forced into cohabitation in the dwindled desert waters and have hybridized so prolifically that 8 per cent of the total minnow population of the Mohave River system is now composed of intergeneric hybrids, or of subspecific intergrades-depending on judgment. Probably on somewhat similar grounds the blind cave characin of Mexico, Anoptichthys jordani, has interbred with its ancestral type, Astyanax fasciatus mexicanus, to produce a complete series of what may with equal propriety be called intergeneric hybrids or subspecific intergrades. Our extensive researches into both phenomena have failed to disclose any essential or consistent distinction between subspecific intergradation and interspecific hybridization.

It is perhaps unfortunate for an orderly taxonomy, or for a pretty scheme of speciation, that the systematic situation is so complex. But neither in detailed taxonomic treatment nor in general speciational theory should we forget the true situation. Arbitrary decisions must often be made, to meet the demands of the Linnaean system of zoological nomenclature, but it is bad science to deny that the decisions are arbitrary. Neither conventionalized views nor subjective subterfugeswhether by the old-line systematist or by the modern speciationist-can transcend the facts, or create a simple "correct" system of taxonomy or a simple theory of speciation out of a situation that is inherently complex. Evolution has been and remains at work:

There appear to be no objective criteria for genera. 